\title{
Hippocampal abnormalities and age in chronic schizophrenia: morphometric study across the adult lifespan
}

N. Pujol, R. Penadés, C. Junqué, I. Dinov, C. H. Y. Fu, R. Catalán, N. Ibarretxe-Bilbao,

N. Bargalló, M. Bernardo, A. Toga, R. J. Howard and S. G. Costafreda

\section{Background}

Hippocampal abnormalities have been demonstrated in schizophrenia. It is unclear whether these abnormalities worsen with age, and whether they affect cognition and function

\begin{abstract}
Aims
To determine whether hippocampal abnormalities in chronic schizophrenia are associated with age, cognition and sociooccupational function.

\section{Method}

Using 3T magnetic resonance imaging we scanned 100 persons aged 19-82 years: 51 were out-patients with stable schizophrenia at least 2 years after diagnosis and 49 were healthy volunteers matched for age and gender. Automated analysis was used to determine hippocampal volume and shape.
\end{abstract}

\section{Results}

There were differential effects of age in the schizophrenia and control samples on total hippocampal volume (group $\times$ age interaction: $\left.F_{(1,95)}=6.57, P=0.012\right)$, with steeper age-related reduction in the schizophrenia group. Three-dimensional shape analysis located the age-related deformations predominantly in the mid-body of the hippocampus. In the schizophrenia group similar patterns of morphometric abnormalities were correlated with impaired cognition and poorer socio-occupational function.

\section{Conclusions}

Hippocampal abnormalities are associated with age in people with chronic schizophrenia, with a steeper decline than in healthy individuals. These abnormalities are associated with cognitive and functional deficits, suggesting that hippocampa morphometry may be a biomarker for cognitive decline in older patients with schizophrenia.

\section{Declaration of interest}

None.
A convergent body of research shows that the hippocampus is involved in the pathophysiology of schizophrenia. ${ }^{1,2}$ Neuroimaging studies have consistently observed reduced hippocampal volume and shape deformities, ${ }^{3-5}$ which have been related to learning and memory impairment. ${ }^{1}$ Moreover, first-episode studies strongly suggest that the decrease in hippocampal grey matter is present at the onset of the disease, ${ }^{3}$ which has often been interpreted as supporting a neurodevelopmental model of schizophrenia. Whether hippocampal loss continues in the chronic phase of schizophrenia has not been clearly established. Hippocampal volume reduction is more marked in patients with chronic illness than in those with a first episode,${ }^{6}$ and correlates with illness duration, ${ }^{7}$ but the findings in longitudinal studies have been mixed. ${ }^{8,9}$ However, previous studies did not include patients over 60 years old, instead basing their conclusions on young and middle-aged patients. As a result of changes during neurodevelopment and psychosis onset, elderly patients with schizophrenia may have decreased brain reserve with increased vulnerability to age-related changes that target the hippocampus, ${ }^{10}$ which may explain these individuals' increased risk of dementia. ${ }^{11}$ In Alzheimer's disease hippocampal atrophy is a strong predictor of worsening cognition and functional impairment. ${ }^{12}$ It remains unclear to what extent hippocampal abnormalities are also linked to functional and cognitive changes in elderly people with schizophrenia.

Three-dimensional morphometric analysis of the hippocampus may be more sensitive to structural abnormalities than volumetric analysis. $^{12}$ By precisely localising small subregional losses, hippocampal shape analysis may be more sensitive to age-related changes and can suggest specific pathological mechanisms underlying hippocampal loss in schizophrenia, of which several have been proposed. ${ }^{1,2}$ We sought to identify the presence of hippocampal morphological abnormalities as a function of age and the impact of such abnormalities on cognitive and functional outcome in people with schizophrenia. Patients with a diagnosis of schizophrenia as well as age- and gender-matched controls were recruited to undergo morphometry of the hippocampus (volume and tri-dimensional shape) to test the following hypotheses:

(a) the schizophrenia group would show a more pronounced effect of age on hippocampal morphometry than the control group;

(b) the effect of ageing on hippocampal volume would not be linear, with more pronounced reductions in older participants resulting in non-linear age trajectories;

(c) hippocampal changes would be related to general cognitive abilities, specifically memory, as well as to functional capacity in older participants.

\section{Method}

Fifty-one patients aged 19-82 years were recruited by the schizophrenia unit at the Hospital Clinic, University of Barcelona (Catalonia, Spain). The diagnosis of schizophrenia was confirmed by an experienced clinician using the Structured Clinical Interview for DSM-IV-TR Axis I Disorders (SCID-I). ${ }^{13}$ Exclusion criteria were an existing diagnosis of organic mental disorder or intellectual disability, a primary diagnosis of disorder due to psychoactive substance use, or psychotic symptom exacerbation or 
electroconvulsive treatment in the previous 6 months. A total of 49 age-matched healthy control participants were recruited from the same catchment area and screened with the non-patient version of the SCID. ${ }^{14}$ Exclusion criteria included: a lifetime history of schizophrenia or other psychotic disorder, bipolar disorder, recurrent depression, or substance dependence or misuse; any of the following Axis II disorders - avoidant, paranoid, schizoid, schizotypal or borderline; a diagnosis of schizophrenia or other psychotic disorder in a first-degree relative; or a history of organic mental disorder, intellectual disability or head injury.

Following a complete description of the study to participants, written informed consent was obtained and protocols were approved by the Human Investigation Committee at the University of Barcelona and the Hospital Clinic of Barcelona.

\section{Clinical and cognitive measures}

A test battery was administered to all participants in a fixed order by an expert neuropsychologist (N.P). The test battery included a global measure of verbal comprehension, reflecting global IQ (the vocabulary subtest of the Wechsler Adult Intelligence Scale Revised, WAIS-III-R), ${ }^{15}$ and a verbal memory domain, the Rey Auditory Verbal Learning Test (RAVLT) ${ }^{16}$ providing memory subdomain scores in learning (RAVLT - Total) and retrieval (RAVLT - Delayed). Demographic details, medical history and medication were captured through a structured interview. In older patients only (50 years or over) we used the Mini-Mental State Examination (MMSE) in its Spanish version and the Functional Activities Questionnaire (FAQ) to measure cognitive and sociooccupational functional status respectively. ${ }^{17,18}$ Symptom severity was assessed in patients at the time of scanning using the Positive and Negative Syndrome Scale (PANSS). ${ }^{19}$

\section{Image acquisition and hippocampal segmentation}

Images were acquired on a $3 \mathrm{~T}$ magnetic resonance imaging (MRI) scanner (Magnetom Trio Tim, Siemens Medical Systems, Germany). A $T_{1}$-weighted structural image was acquired for each participant with magnetisation prepared rapid gradient echo (MPRAGE) three-dimensional (3D) protocol: time to repetition $2300 \mathrm{~ms}$, time to echo $2.98 \mathrm{~ms}$, 240 slices, slice thickness $1 \mathrm{~mm}$, field of view $256 \times 256 \mathrm{~mm}^{2}$, matrix size $256 \times 256$. Automated hippocampal segmentation was performed using a pattern recognition algorithm validated to extract hippocampi automatically from the high-resolution images. This algorithm computes whether a voxel belongs to right or left hippocampus based on its location and properties extracted from its local neighbourhood, such as intensities, gradients and curvatures across different scales. ${ }^{20}$ Segmented outputs of the algorithm have been shown to have good agreement with independent hippocampal segmentations produced by human experts, ${ }^{20}$ based on a standardised segmentation protocol (http://resource.loni.usc.edu/ resources/downloads/research-protocols/segmentation/hippocampusdelineation/). Total hippocampal volume was computed by adding the segmented hippocampi volumes. Total intracranial volume and total grey-matter volume were computed with Freesurfer (http://surfer.nmr.mgh.harvard.edu/) run on Linux (Cent0S-4 x86_64) and used to normalise hippocampal measures. ${ }^{21}$ For shape analysis an initial 3D mesh representation of each hippocampus was constructed based on the segmented images. Direct hippocampal mapping was then used to map the segmented hippocampi into $3 \mathrm{D}$ shape representations robust to variations in orientation or position of the hippocampus across participants. ${ }^{22}$ This procedure allows the determination of the radial distance or 'thickness' of the hippocampus at a particular point in its surface. A reduction in radial distance can be interpreted as evidence of atrophy. Automated segmentation and volumetric and shape analysis were performed in Laboratory of Neuroimaging (LONI) Pipeline following a validated protocol. ${ }^{12}$ The $T_{1}$ images, hippocampal segmentation outputs and resulting shape analysis were submitted to manual quality control, inclusive of operationalised visual quality checks.

\section{Statistical analysis}

General linear modelling (GLM) was used to study the effects of age across diagnostic groups (schizophrenia $v$. control) on normalised hippocampal volume and shape (normalised radial thickness), with gender as covariate of no interest. The presence of differential age effects between the patient and control groups was assessed through the statistical significance of a clinical groupage interaction term. The presence of non-linear effects was assessed by means of analysis of variance (ANOVA) tests of nested models with successive addition of linear, quadratic and cubic effects. The resulting model fits were compared using $F$-tests to determine significant increases in explanatory power justifying the addition of new terms. Given the evidence that low educational attainment is a risk factor for decreased hippocampal volume in later life, ${ }^{23}$ we studied the effects of years of education as a potential confounder. Mediation effects were studied using the Sobel test. ${ }^{24}$ The GLM, ANOVA and mediation analyses were run with the $\mathrm{R}$ statistical software package. To map the association between hippocampal shape and the variables of interest, independent models analogous to the ones described above for volume were fitted at each vertex of the hippocampus shape (2000 vertices in total for each statistical map). Multiple comparisons correction was performed by permutation testing using a set-level statistic, namely the number of vertices whose $P$ value for the covariate of interest survived an initial uncorrected threshold of $P<0.01 .^{25}$ The experimental statistic ( $F$ or $t$ ) obtained from the observed map was then compared with a permutation-driven distribution. This distribution was generated under the null hypothesis of no association between local radial distance and the variables of interest by permuting the values of the predicted variable across participants, refitting the model with the permuted data and re-computing the statistic for the covariate of interest. ${ }^{26}$ We used 10000 iterations of this permutation procedure to test the overall statistical significance of the statistical maps. The corrected $P$ value measures whether the whole pattern of association can be declared statistically significant. As $T_{1}$ imaging at $3 \mathrm{~T}$ strength does not allow sufficient resolution to resolve hippocampal subfields, the attribution of atrophy findings to specific subfields is approximate, based on previously published subfield atlases. ${ }^{27-29}$

\section{Results}

Patient and control groups were well matched for gender and age. The age range of participants covered the adult lifespan (19-82 years). Those in the schizophrenia group were symptomatically stable (no acute episode in the past 6 months) and had been diagnosed with schizophrenia for a minimum of 2 years before scanning (mean 21.4 years). Three patients had a late onset of their illness (one at age 50 years, two at age 42 years). A complete description of the demographic, cognitive and clinical characteristics of the sample is provided in Table 1 and in online Table DS1.

\section{Effect of age on hippocampal volume and shape}

There were differential effects of age on total hippocampal volume in the schizophrenia and control samples as indicated by a 


\begin{tabular}{|c|c|c|c|c|}
\hline & $\begin{array}{l}\text { Schizophrenia group } \\
\qquad(n=51)\end{array}$ & $\begin{array}{l}\text { Control group } \\
\qquad(n=49)\end{array}$ & Comparison & $P$ \\
\hline \multicolumn{5}{|l|}{ Demographic factors } \\
\hline Age, years: mean (s.d.) & $48.9(17.9)$ & $52.8(17.2)$ & $t(98)=1.12$ & 0.268 \\
\hline Female gender, $n$ & 23 & 23 & $\chi^{2}(1)=0.034$ & 0.506 \\
\hline Education, years: mean (s.d.) & $11.4(5.2)$ & $13.3(4.5)$ & $t(98)=1.88$ & 0.063 \\
\hline \multicolumn{5}{|l|}{ MRI volume, $\mathrm{cm}^{3}$ : mean (s.d.) } \\
\hline Right hippocampus & $2.4(0.4)$ & $2.6(0.3)$ & $t(98)=1.58$ & 0.117 \\
\hline Left hippocampus & $2.8(0.4)$ & $2.9(0.4)$ & $t(98)=1.78$ & 0.078 \\
\hline \multicolumn{5}{|l|}{ Clinical factors } \\
\hline PANSS score (total): mean (s.d.) & $58.3(18.0)$ & NA & & \\
\hline Illness duration, years: mean (s.d.) & $21.4(15.8)$ & NA & & \\
\hline
\end{tabular}

statistically significant age $\times$ group interaction effect $(F=6.57$, d.f.(1) $=1$, d.f. $(2)=95, P=0.012)$. Linear age effects fitted to the two groups separately revealed a steeper decline of hippocampal volume with age (more negative slope) in the schizophrenia group relative to controls (Fig. 1). Given previous evidence of non-linear structural changes, ${ }^{30}$ as well as of non-linear age-related cognitive decline in older patients with schizophrenia, ${ }^{31}$ we tested the presence of non-linear effects between age and hippocampal volume by comparing the above model (which assumed a linear effect of age) with models incorporating additional quadratic and cubic associations between age and hippocampal volume. The model with a quadratic relationship provided the best explanation of the relationship between age and hippocampal volume in schizophrenia, with a significantly better fit to the data than the model based on linear decline only (ANOVA for model

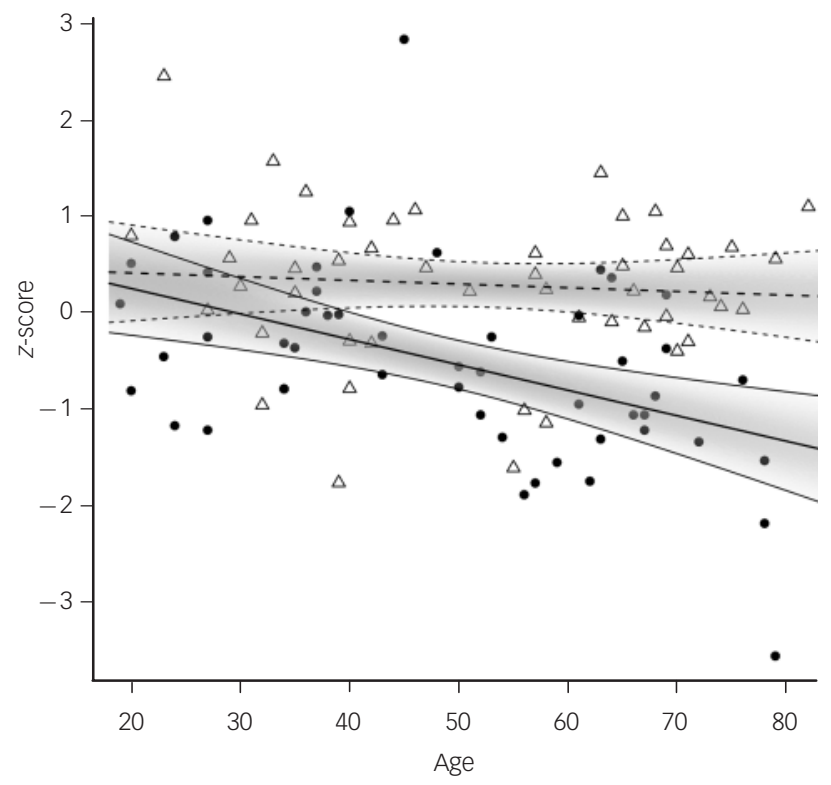

Fig. 1 Hippocampal volume as a function of age in schizophrenia and control groups. Total hippocampal volume has a steeper decline as a function of age in patients with schizophrenia (solid circles; solid line is best linear fit) than in the healthy control group (triangles; dashed line is best linear fit). Hippocampal volume has been normalised to $z$ scores, adjusted for gender and intracranial volume; the shaded area corresponds to the $95 \%$ confidence interval for the fit. comparison $F=5.21$, d.f. $(1)=1$, d.f. $(2)=47, \quad P=0.027)$. The model with cubic effects was not significantly better than the linear $(F=2.58$, d.f. $(1)=2$, d.f. $(2)=46, P=0.086)$ or quadratic $(F=0.04$, d.f. $(1)=1$, d.f. $(2)=46, P=0.807)$ models. The quadratic relationship suggests a period of accelerating decline in patients with schizophrenia relative to healthy controls after the age of 50 years (see online Fig. DS1).

As educational attainment is an independent predictor of hippocampal volume, we conducted a mediation analysis to investigate whether the effect of schizophrenia diagnosis on hippocampal volume could be explained by less education in the patient group. Education was not a statistically significant mediator of the relationship between schizophrenia and hippocampal volume (Sobel test $Z=1.40, P=0.162$ ), suggesting that at least some of the effects of the illness were independent of its effects on education. Similarly, adjusting by years of education did not alter the finding of a significant age $\times$ group interaction effect on hippocampal volume, whereby the schizophrenia group demonstrated a steeper decline with age, even when reduced education was taken into account (differential group $\times$ age effect in the adjusted model: $F=6.14$, d.f. $(1)=1$, d.f. $(2)=87, P=0.015)$.

A sensitivity analysis was also conducted to assess the robustness of the above findings to operationally defined outliers (the analysis is described in the online supplement Method section); although the finding of a steeper linear decline in schizophrenia was robust to the deletion of extreme measurements, the presence of accelerating decline in older patients (significant quadratic effects) was not, and should therefore be treated with caution. Similarly, there was a statistically significant linear relationship between disease duration and hippocampal volume, whereas non-linear effects failed to reach significance. Finally, the analysis was repeated after normalisation for total grey-matter volume (instead of total intracranial volume). Again, there were differential linear effects of age on hippocampal volume in the schizophrenia group relative to controls (age $\times$ group interaction: $F=5.85$, d.f. $(1)=1$, d.f. $(2)=95, P=0.017$ ), suggesting that this association was not due to generalised effects of age and schizophrenia diagnosis on cortical atrophy, but reflected a specific hippocampal effect.

Three-dimensional shape analysis was employed to investigate the regional distribution of effects within the hippocampus. This analysis revealed areas of age-related accelerated atrophy in schizophrenia, compared with healthy controls, in the mid-body of the right hippocampus (approximating to the cornu ammonis or CA1, CA2/3), and to a lesser extent into its tail (see online Fig. DS2(a), $P<0.001$, permutation-corrected for 
multiple comparisons). Areas of accelerated atrophy in the left hippocampus mirrored this pattern, but were more localised (corrected $P=0.035$ ).

\section{Cognitive and clinical function}

In the schizophrenia group decreased hippocampal volume was associated with worse scores on general measures of cognitive function. Patients with smaller hippocampi performed worse in the WAIS-III-R vocabulary subtest $(t=2.45$, d.f. $=46, P=0.018)$. In older patients, those with lower hippocampal volume also had lower MMSE scores $(t=2.65$, d.f. $=19, P=0.016)$ and the association between smaller hippocampi and worse sociooccupational function approached significance (FAQ score: $t=-1.97$, d.f. $=18, P=0.064)$. In these patients there was a strong correlation between FAQ score and vocabulary $(t=-3.54$, d.f. $=18, P<0.001)$ as well as between FAQ and MMSE scores $(t=-5.84$, d.f. $=18, P<0.001)$ but not between FAQ score and age $(t=0.84$, d.f. $=18, \quad P=0.411)$, suggesting that sociooccupational function might be mediated by general cognitive performance, of which hippocampal volume is a strong correlate and (possibly) substrate. A causal mediation analysis of the influence of the MMSE score as mediator between hippocampal volume and FAQ was statistically significant (Sobel test, $Z=-2.22, P=0.026$ ), supporting the notion that a substantial part of the effects of hippocampal volume on functional ability is mediated by general cognitive performance. In contrast, specific measures of memory encoding and retrieval were not associated with volume (RAVLT - Total $t=1.52$, d.f. $=46, \quad P=0.136$; RAVLT - Delayed $t=0.579$, d.f. $=46, P=0.565$ ). There was also no significant association of hippocampal volume with the presence of current psychotic symptoms (PANSS positive symptoms $t=0.089$, d.f. $=42, P=0.93$; PANSS negative symptoms $t=-0.108$, d.f. $=42, P=0.914$; PANSS general pathology $t=-0.23$, d.f. $=42, P=0.819)$ or antipsychotic dosage $(t=-0.543$, d.f. $=49$, $P=0.59$ ).

Morphological shape analysis revealed that MMSE score in older patients with schizophrenia was associated with a distributed pattern of changes within the hippocampus (Fig. DS2(b); $P=0.013$ for right hippocampus, $P=0.032$ for left hippocampus, permutation-corrected for multiple comparisons). This pattern was similar to that expressing accelerated atrophy in schizophrenia (Fig. DS2(a)), but with further extension to right hippocampal head and left hippocampal tail. Finally, shape analysis also revealed that socio-occupational function (FAQ score) was associated with changes in hippocampi that were similar to those associated with MMSE score (Fig. DS2(c); $P=0.028$ for right hippocampus, $P=0.066$ for left hippocampus, permutationcorrected for multiple comparisons). The similarity in the patterns reinforces the possibility that these hippocampal regions are subserving aspects of cognition that are relevant to effective functional ability.

\section{Discussion}

Our study demonstrated a steeper reduction of hippocampal volume during adult life in schizophrenia than in healthy ageing. Volume loss in schizophrenia may also accelerate after age 50 years, although this later finding was not robust to outlier effects and should therefore be treated with caution pending confirmation. Hippocampal atrophy is strongly associated with global cognitive and socio-occupational functional impairments in older patients, but not with clinical symptoms or specific memory scores. Hippocampal volume loss during the first years of disease is one of the most consistent findings in schizophrenia research. ${ }^{3,5}$ In people with chronic schizophrenia hippocampal volume reduction appears to progress further than in those with first-episode disorder, ${ }^{6}$ and correlates with illness duration. ${ }^{7}$ Although 2-year rescanning has failed to identify changes in the hippocampus of patients with chronic illness, ${ }^{8}$ a longer-term study by Koolschijn et al, ${ }^{9}$ with an average follow-up period of 5 years, demonstrated ongoing hippocampal volume loss at a constant rate of atrophy over time in chronic schizophrenia. This longer-term study, however, did not find evidence for acceleration of volume loss with age in patients, perhaps because its findings were based almost exclusively on young and middle-aged patients. ${ }^{9}$ Our study, which included a sample of patients at least 2 years after initial diagnosis and covered the adult lifespan into old age, also indicates that hippocampal volume reduction does occur in chronic stages of the illness, develops over decades and may accelerate in older patients ( $>50$ years). These effects in the hippocampus are not explained by the global effect of ageing on the cortex, and may therefore be at least partly specific to this brain region.

\section{Hippocampus in older patients}

One interpretation of our findings is that an early neurodevelopmental mechanism may be followed in later years by a subsequent component of possibly neurodegenerative aetiology, at least in a subgroup of patients. Injury during neurodevelopment and first-episode psychosis to the hippocampus may result in decreased brain reserve, which manifests itself as increased vulnerability to neurodegenerative processes in old age or even by increased negative effects of normal ageing processes. ${ }^{10}$ Older patients with schizophrenia have a high risk of dementia, ${ }^{11}$ and although post-mortem tissue studies have failed to demonstrate typical Alzheimer's disease or Lewy body neuropathology in schizophrenia, ${ }^{11}$ new MRI evidence does suggest excessive neurodegeneration in older patients. Schuster et al have demonstrated significant reductions in grey-matter volume in elderly patients with schizophrenia compared with healthy controls in a network of regions inclusive of medial temporal cortex. $^{32}$ In addition, Prestia et al reported greater left hippocampal and amygdalar atrophy in elderly patients with schizophrenia relative to patients with Alzheimer's disease (ages 60-78 years). ${ }^{33}$ Intriguingly, Rapp et al recently demonstrated a significant association between neuritic plaques and hippocampal neurofibrillary tangles and dementia severity in geriatric patients with schizophrenia but without a neuropathological diagnosis of Alzheimer's disease. ${ }^{34}$ Finally, Casanova et al suggested a pathological substrate of limbic tauopathy that may be seen in up to a third of patients with early-onset schizophrenia. ${ }^{35}$ Although the pathology of the dementing process has yet to be identified, our findings suggest that hippocampal changes may have an important role.

Our data point to a steeper gradient of hippocampal decline with age in people with the illness, which suggests a process of neurodegeneration associated with schizophrenia later in life. The aetiology of this process, however, could still be lower cognitive reserve in people with schizophrenia relative to age-matched controls. One determinant of cognitive reserve is education, and there is some initial evidence that vulnerability acquired earlier in life for hippocampal atrophy because of low education may express itself only later in life. ${ }^{23}$ The finding of a steeper gradient in older patients, however, survived adjustment for education. Moreover, the mediation effects of education on the relationship between hippocampal volume and diagnosis were not statistically significant. This suggests that at least some of the effects of the 
illness on hippocampal volume in old age are independent of low educational levels earlier in the patient's life.

\section{Hippocampus deformations}

Hippocampal shape analysis was conducted to locate precisely the subregional pattern of age-related atrophy in schizophrenia compared with a healthy control group. In the right hippocampus there was evidence of accelerated shrinkage in the mid-body (approximately identified as CA1 and CA2,3) and to a lesser extent in the tail. In the left hippocampus age-related deformities were more limited, but also predominantly located in the mid-body with some extension towards anterior regions (identified as CA1 and subiculum). Bilaterally, then, the strongest age effects were seen predominantly in mid-body regions of the hippocampus, in a similar distribution to the changes described in previous studies of hippocampal shape in patients with first-episode and chronic disease. ${ }^{4,5}$ These subregional effects also overlap with the hippocampal regions previously linked to the functional changes involved in schizophrenia onset. $^{2}$ In addition, morphometric studies of the hippocampus in depression have highlighted atrophy in more posterior regions. ${ }^{36,37}$ This pattern of abnormalities is distinct from the one typically observed in Alzheimer's disease progression, which has an anterior-to-posterior progression with onset in the anterior hippocampus (head), as determined in follow-up studies of cognitively normal samples, ${ }^{28}$ and of individuals with mild cognitive impairment who subsequently developed Alzheimer's disease. ${ }^{12}$ Direct comparisons across illnesses, however, will be required to establish whether these effects are truly different. Our findings add to the neuropathological evidence reviewed above, suggesting that although the hippocampus may be an important substrate of schizophrenia-related dementia, the mechanisms involved are likely to be different from those present in Alzheimer's disease dementia. In our sample, ageing in schizophrenia was associated with more extensive right-sided hippocampal reduction. It has been suggested that right hippocampal atrophy preferentially reflects the chronic effects of schizophrenia, ${ }^{6,38}$ whereas left hippocampal atrophy would be more evident in very early stages of the illness. ${ }^{39}$ Our findings are consistent with these previous results, although we were unable to test this laterality difference directly as all the participants in our schizophrenia sample were recruited at least 2 years after diagnosis.

\section{Hippocampal atrophy and cognitive and clinical variables}

There was a strong correlation between hippocampal morphometric abnormalities and measures of general cognition (vocabulary and MMSE score) in older patients with schizophrenia. Since these are measures of general cognitive functioning related to cortical functions, not just memory, this correlation may reflect the fronto-hippocampal disconnection documented in schizophrenia rather than the sole effects of hippocampal atrophy. ${ }^{40}$ Testing this hypothesis in future studies will require assessment with instruments sensitive to frontal dysfunction, rather than general tools such as the MMSE. Contradicting our a priori hypothesis, however, we failed to find a significant association between decreased total hippocampal volume and specific memory deficits in patients (verbal encoding and retrieval), ${ }^{1}$ which again is distinct from the findings in Alzheimer's disease in which hippocampal volume is strongly correlated with memory scores. ${ }^{12,41}$ A necessary caveat is that this negative finding might be due to limited power rather than a true lack of association.

There was a significant relationship between hippocampal shape and poor socio-occupational function as measured by the FAQ scores of elderly participants in the schizophrenia group, but no significant association was found between FAQ and age. The pattern of changes associated with poor FAQ score was similar to that associated with impaired general cognition (MMSE score), strongly suggesting that poor functioning in this sample is mediated by cognition. ${ }^{42,43}$ Taken together, these findings point to a role for hippocampal abnormalities as a biomarker for general cognitive decline and functional impairment in late-life schizophrenia.

Regarding the potential impact of antipsychotic medication on regional brain structure, we did not find a significant association between chlorpromazine equivalent dosages and hippocampal volume. Longitudinal studies in humans have contradictory results, ${ }^{44,45}$ which may be due to the difficulty of separating the effects of illness and its treatment. Animal evidence suggests that antipsychotic toxicity does not affect hippocampal cortices. ${ }^{46}$

\section{Limitations}

Although the cross-sectional findings presented here show robust evidence of association between age and worsening hippocampal abnormalities, definite confirmation requires longitudinal rescanning. In particular, the possibility of accelerated abnormalities in elderly patients, which may result in increased risk of dementia, needs to be confirmed in a longitudinal sample enriched with older patients to ensure sufficient power. Pending this confirmation, an alternative explanation of our findings is that patients with smaller hippocampi from disease onset may be more predisposed to chronic illness, and therefore overrepresented in the older patient group. Similarly, we cannot exclude an increased effect of vascular risk factors in older patients relative to controls, which could also result in reduced hippocampal volume. To our knowledge, the long-term impact of metabolic and other cardiovascular risk factors on brain structure has not been previously reported in schizophrenia. On the other hand, older patients and control groups are a selected (generally healthier) sample of all patients and controls, simply by virtue of surviving into old age.

The analysis of length of education in patients and controls in older and younger participants suggests that early deprivation was not a confounder through cohort effects (see online supplement Results section). However, the presence of unmeasured confounders, including cohort effects, cannot be excluded in an observational design. Finally, we found no evidence of a relationship between hippocampus volume and current antipsychotic dose, but it is possible that some of the differences in the atrophy rates of the patient and control groups are due to the lifetime use of antipsychotics, which we could not measure, rather than primary effects of the illness.

\section{Study implications}

People with schizophrenia experience hippocampal atrophy in excess of that observed in healthy ageing, with some evidence of further acceleration in older age. Hippocampal atrophy has a high degree of relevance for the expression of cognitive and socio-occupational deficits, particularly in older adults. Therefore, hippocampal atrophy may have a potential role as a biomarker for decline in late-life schizophrenia, and may be a key to clarifying the causes of the increased dementia risk in this population. Future studies, especially longitudinal research in geriatric samples, are needed to confirm these findings. 


\section{Funding}

The study was supported by the Emili Letang award from the Hospital Clinic of Barcelona to N.P. and grants from the Instituto de Salud Carlos III (FIS PI 07/0258) and Rio Hortega to N.P. The Pere Pons Foundation and the Spanish Ministry of Education supplied individual mobility grants to N.P. S.G.C. is supported by a National Institute of Health Research Academic Clinical Lecturership. Funding sources had no role in the design or conduct of the study or in the analysis and interpretation of the data.

Núria Pujol, PhD, Department of Psychiatry and Clinical Psychobiology, University of Barcelona, Institut d'Investigacions Biomèdiques August Pi i Sunyer (IDIBAPS), Barcelona, Clinical Institute of Neurosciences (ICN), Hospital Clinic, Barcelona, Spain Rafael Penadés, PhD, Department of Psychiatry and Clinical Psychobiology, University of Barcelona, IDIBAPS, Barcelona, ICN, Hospital Clinic, Barcelona, Centre for Biomedical Research on Mental Health (CIBERSAM), Instituto de Salud Carlos III, Barcelona, Spain; Carme Junqué, PhD, Department of Psychiatry and Clinical Psychobiology, University of Barcelona, IDIBAPS, Barcelona, Spain; Ivo Dinov $\mathrm{PhD}$, Laboratory of Neuroimaging, University of California, Los Angeles, USA; Cynthia H. Y. Fu, MD, PhD, Department of Old Age Psychiatry, Institute of Psychiatry, King's College London, UK; Rosa Catalán, MD, PhD, Department of Psychiatry and Clinical Psychobiology, University of Barcelona, IDIBAPS, Barcelona, ICN, Hospital Clinic, Barcelona, CIBERSAM, Instituto de Salud Carlos III, Barcelona, Spain: Naroa Ibarretxe-Bilbao, PhD, Department of Methods and Experimenta Psychology, Faculty of Psychology and Education, University of Deusto, Bilbao, Spain Púria Bargalló, MD, PhD, IDIBAPS, Barelona, ICN, Barcelona, CIBERSAM, Instito Núria Bargallo, MD, PhD, IDIBAPS, Barcelona, ICN, Barcelona, CIBERSAM, Instit de Salud Carlos III, Barcelona, Spain; Miquel Bernardo, MD, PhD, Departmen of Psychiatry and Clinical Psychobiology, University of Barcelona, IDIBAPS, Barcelona, ICN, Barcelona, CIBERSAM, Instituto de Salud Carlos III, Barcelona, Spain Arthur Toga, PhD, Laboratory of Neuroimaging, University of California, Los Angeles, USA; Robert J. Howard, MRCPsych, Sergi G. Costafreda, MD, PhD, Department of Old Age Psychiatry, Institute of Psychiatry, King's College London, UK

Correspondence: Dr Sergi G. Costafreda, Institute of Psychiatry, De Crespigny Park, PO BOX 70, London SE5 8AF, UK. Email: sergi.1.costafreda@kcl.ac.uk

First received 16 Oct 2013, final revision 27 Feb 2014, accepted 1 May 2014

\section{References}

1 Tamminga CA, Stan AD, Wagner AD. The hippocampal formation in schizophrenia. Am J Psychiatry 2010; 167: 1178-93.

2 Small SA, Schobel SA, Buxton RB, Witter MP, Barnes CA. A pathophysiological framework of hippocampal dysfunction in ageing and disease. Nat Rev Neurosci 2011; 12: 585-601.

3 Adriano F, Caltagirone C, Spalletta G. Hippocampal volume reduction in first-episode and chronic schizophrenia: a review and meta-analysis. Neuroscientist 2012; 18: 180-200

4 Velakoulis D, Stuart GW, Wood SJ, Smith DJ, Brewer WJ, Desmond P, et al. Selective bilateral hippocampal volume loss in chronic schizophrenia. Biol Psychiatry 2001; 50: 531-9.

5 Narr KL, Thompson PM, Szeszko P, Robinson D, Jang S, Woods RP, et al Regional specificity of hippocampal volume reductions in first-episode schizophrenia. Neuroimage 2004; 21: 1563-75.

6 Velakoulis D, Wood SJ, Wong MT, McGorry PD, Yung A, Phillips L, et al. Hippocampal and amygdala volumes according to psychosis stage and diagnosis: a magnetic resonance imaging study of chronic schizophrenia, first-episode psychosis, and ultra-high-risk individuals. Arch Gen Psychiatry 2006; 63: 139-49.

7 Chakos MH, Schobel SA, Gu H, Gerig G, Bradford D, Charles C, et al. Duration of illness and treatment effects on hippocampal volume in male patients with schizophrenia. Br J Psychiatry 2005; 186: 26-31.

8 Wood SJ, Velakoulis D, Smith DJ, Bond D, Stuart GW, McGorry PD, et al. A longitudinal study of hippocampal volume in first episode psychosis and chronic schizophrenia. Schizophr Res 2001; 52: 37-46.

9 Koolschijn PC, van Haren NE, Cahn W, Schnack HG, Janssen J, Klumpers F, et al. Hippocampal volume change in schizophrenia. J Clin Psychiatry 2010 71: $737-44$

10 Arnold SE. Contributions of neuropathology to understanding schizophrenia in late life. Harv Rev Psychiatry 2001; 9: 69-76.

11 Arnold SE, Trojanowski JQ, Gur RE, Blackwell P, Han L, Choi C. Absence of neurodegeneration and neural injury in the cerebral cortex in a sample of elderly patients with schizophrenia. Arch Gen Psychiatry 1998; 55: 225-32.

12 Costafreda SG, Dinov ID, Tu Z, Shi Y, Liu CY, Kloszewska I, et al. Automated hippocampal shape analysis predicts the onset of dementia in mild cognitive impairment. Neuroimage 2011; 56: 212-9.

13 First MB, Spitzer RL, Gibbon M, Williams JBW. Structured Clinical Interview for DSM-IV-TR Axis I Disorders, Research Version, Patient Edition. (SCID-I/P) Biometrics Research, New York State Psychiatric Institute, 2002.
14 First MB, Spitzer RL, Gibbon M, Williams JBW. Structured Clinical Interview for DSM-IV-TR Axis I Disorders, Research Version, Non-patient Edition (SCID-I/ NP). Biometrics Research, New York State Psychiatric Institute, 2002.

15 wechsler D. Wechsler Adult Intelligence Scale - Revised. TEA Ediciones, 1981.

16 Rey A. L'Examen Clinique en Psychologie. Press Universitaire de France, 1964.

17 Lobo A, Saz P, Marcos G, Día JL, de la Cámara C, ventura T, et al. Revalidation and standardization of the cognition mini-exam (first Spanish version of the Mini-Mental Status Examination) in the general geriatric population. Med Clin (Barc) 1999; 112: 767-74.

18 Pfeffer RI, Kurosaki TT, Harrah CH, Chance JM, Filos S. Measurement of functional activities in older adults in the community. J Gerontol 1982; 37 323-9.

19 Kay SR, Fiszbein A, Vital-Herne M, Fuentes LS. The Positive and Negative Syndrome Scale - Spanish adaptation. J Nerv Ment Dis 1990; 178: 510-7.

20 Morra JH, Tu Z, Apostolova LG, Green AE, Avedissian C, Madsen SK, et al. Validation of a fully automated $3 \mathrm{D}$ hippocampal segmentation method using subjects with Alzheimer's disease mild cognitive impairment, and elderly controls. Neuroimage 2008; 43: 59-68.

21 Fischl B. Freesurfer. Neuroimage 2012; 62: 774-81

22 Shi Y, Thompson PM, de Zubicaray GI, Rose SE, Tu Z, Dinov I, et al. Direct mapping of hippocampal surfaces with intrinsic shape context. Neuroimage 2007; 37: 792-807.

23 Noble KG, Grieve SM, Korgaonkar MS, Engelhardt LE, Griffith EY, Williams LM, et al. Hippocampal volume varies with educational attainment across the lifespan. Front Hum Neurosci 2012; 6: 307.

24 Sobel ME. Asymptotic confidence intervals for indirect effects in structural equation models. Sociol Method 1982; 13: 290-312.

25 Friston KJ, Holmes A, Poline JB, Price CJ, Frith CD. Detecting activations in PET and fMRI: levels of inference and power. Neuroimage 1996; 4 : 223-35.

26 Anderson M, Robinson J. Permutation tests for linear models. Aust NZ J Stat 2001: 43: 75-88.

27 West MJ. Stereological studies of the hippocampus: a comparison of the hippocampal subdivisions of diverse species including hedgehogs, laboratory rodents, wild mice and men. Prog Brain Psychiatry Res 1998; 84: 75-88.

28 Csernansky JG, Wang L, Swank J, Miller JP, Gado M, McKeel D, et al. Preclinical detection of Alzheimer's disease: hippocampal shape and volume predict dementia onset in the elderly. Neuroimage 2005; 25 : 783-92.

29 Yushkevich PA, Avants BB, Pluta J, Das S, Minkoff D, Mechanic-Hamilton D, et al. A high-resolution computational atlas of the human hippocampus from postmortem magnetic resonance imaging at 9.4T. Neuroimage 2009; 44 385-98

30 DeLisi LE, Sakuma M, Ge S, Kushner M. Association of brain structura change with the heterogeneous course of schizophrenia from early childhood through five years subsequent to a first hospitalization. Schizophr Res 2001; 83: 13-36.

31 Friedman Jl, Harvey PD, Coleman T, Moriarty PJ, Bowie C, Parrella M, et al. Six-year follow-up study of cognitive and functional status across the lifespan in schizophrenia: a comparison with Alzheimer's disease and normal aging. Am J Psychiatry 2001; 158: 1441-8.

32 Schuster C, Schuller AM, Paulos C, Namer I, Pull C, Danion JM, et al. Gray matter volume decreases in elderly patients with schizophrenia: a voxel-based morphometry study. Schizophr Bull 2012; 38: 796-802.

33 Prestia A, Boccardi M, Galluzzi S, Cavedo E, Adorni A, Soricelli A, et al. Hippocampal and amygdalar volume changes in elderly patients with Alzheimer's disease and schizophrenia. Psychiatry Res 2011; 192: 77-83.

34 Rapp MA, Schnaider-Beeri M, Purohit DP, Reichenberg A, McGurk SR, Haroutunian V, et al. Cortical neurotic plaques and hippocampal neurofibrillary tangles are related to dementia in elderly schizophrenia patients. Schizophr Res 2010; 116: 90-6.

35 Casanova MF, Stevens JR, Brown R, Royston C, Bruton C. Disentangling the pathology of schizophrenia and paraphrenia. Acta Neuropathol 2002; 103: $313-20$.

36 Posener JA, Wang L, Price JL, Gado MH, Province MA, Miller Ml, et al. High-dimensional mapping of the hippocampus in depression. Am J Psychiatry 2003; 160: 83-9.

37 Cole J, Toga AW, Hojatkashani C, Thompson P, Costafreda SG, Cleare AJ et al. Subregional hippocampal deformations in major depressive disorder. J Affect Disord 2010; 126: 272-7.

38 Velakoulis D, Pantelis C, McGorry PD, Dudgeon P, Brewer W, Cook M, et al. Hippocampal volume in first-episode psychoses and chronic schizophrenia: 
a high-resolution magnetic resonance imaging study. Arch Gen Psychiatry 1999; 56: 133-41.

39 Pantelis D, Velakoulis J, Suckling PD, McGorry LJ, Phillips AR, Yung SJ, et al. Left medial temporal volume reduction occurs during the transition from high-risk to first-episode psychosis. Schizophr Res 2000; 41: 35.

40 Lipska BK, Weinberger DR. A neurodevelopmental model of schizophrenia: neonatal disconnection of the hippocampus. Neurotox Res 2002; 4: 469-75.

41 Thompson PM, Hayashi KM, De Zubicaray GI, Janke AL, Rose SE, Semple J, et al. Mapping hippocampal and ventricular change in Alzheimer disease. Neuroimage 2004; 22: 1754-66.

42 Penadés R, Boget $\mathrm{T}$, Catalán R, Bernardo M, Gastó C, Salamero M. Cognitive mechanisms, psychosocial functioning, and neurocognitive rehabilitation in schizophrenia. Schizophr Res 2003; 63: 219-27.
43 Green MF. Cognitive impairment and functional outcome in schizophrenia and bipolar disorder. J Clin Psychiatry 2006; 67: e12.

44 Panenka WJ, Khorram B, Barr AM, Smith GN, Lang DJ, Kopala LC, et al. A longitudinal study on the effects of typical versus atypical antipsychotic drugs on hippocampal volume in schizophrenia. Schizophr Res 2007; 94 288-92.

45 Ebdrup BH, Skimminge A, Rasmussen $\mathrm{H}$, Aggernaes B, Oranje B, Lublin $\mathrm{H}$, et al. Progressive striatal and hippocampal volume loss in initially antipsychotic-naive, first-episode schizophrenia patients treated with quetiapine: relationship to dose and symptoms. Int I Neuropsychopharmacol 2011; 14: 69-82.

46 Vernon AC, Natesan S, Modo M, Kapur S. Effect of chronic antipsychotic treatment on brain structure: a serial magnetic resonance imaging study with ex vivo and postmortem confirmation. Biol Psychiatry 2011; 69: 936-44.

\section{reflection}

\section{W. Beers, A Mind That Found Itself}

\section{Pippa Jones}

In 1900, when he was 24, Clifford Whittingham Beers was first confined to a private mental hospital for depression and paranoia after a suicide attempt. He experienced delusions and heard frightening voices. Given that the hospital was in New Haven, Connecticut, a prosperous state, one would hope that the care was of a high standard but regrettably that was not the case.

Beers described his experiences of a total of 3 years in two private mental institutions and the state mental hospital in his groundbreaking book, A Mind That Found Itself, published in 1908. I myself have been hospitalised on three occasions, from 3 weeks' to 3 months' duration, and cannot imagine the horror of spending 3 years in hospital. Beers describes the importance of relatives and friends maintaining contact and I can echo this, visiting hours being the best part of the day. In Chapter XI, the author refers to the stigma of mental illness and one can only say that although reduced, this is still strong today.

I was somewhat taken aback by the lack of context in the book; Beers seems totally removed from anything on the national or world stage going on around him. At one point he searches in vain for newspapers from June 1900 to ascertain whether anything that set his depression off was reported. Another thing that surprised me was the absence of mention of other patients, as I have always enjoyed talking to other people on the ward. However, Beers was prepared to get into fights on behalf of one friend and he seemed to think that this conduct was justified if he verbally warned the staff first. It is interesting, 105 years after publication, that we have recently had a debate about controversial restraint techniques.

Beers describes undesirable equipment such as the muff and his first night in a strait-jacket, after which his conservator effected his transfer to the state hospital. He talked incessantly, he writes. The staff are still wanting: 'It (the curve representing my return to normality) was kept violently fluctuating ... by the impositions of those in charge of me, induced sometimes, I freely admit, by deliberate and purposeful transgressions of my own'. Medication is hardly mentioned at all, in stark contrast to the 21st century. Beers acknowledges the importance of exercise and, although he writes of his interest in art following his discharge in September 1903, this wanes and he returns to his earlier job. It made me think of the many classes I enjoyed when I was in hospital, from creative writing to relaxation, some of which I still practise. The author also refers to the chapel and I, too, found great solace in attending services and talking to the chaplaincy staff.

Beers won the support of the medical profession and other people of influence to reform the treatments of those with mental health conditions. A year after the publication of his book, he founded the National Committee for Mental Hygiene, now known as Mental Health America. 